\title{
Imaging of Rough Surfaces by Near-field Measurement
}

\author{
Wei Chien, ${ }^{1}$ Chien-Ching Chiu, ${ }^{2 *}$ Po-Hsiang Chen, ${ }^{2}$ Hao Jiang, ${ }^{3}$ and Shun-Jie Chan ${ }^{2}$ \\ ${ }^{1}$ Department of Electric and Information Engineering, Beibu Gulf University, Qinzhou 535011, China \\ ${ }^{2}$ Department of Electrical Engineering, Tamkang University, Tamsui 251301, Taiwan \\ ${ }^{3}$ School of Engineering, San Francisco State University, San Francisco, CA 94117-1080, USA
}

(Received December 31, 2020; accepted April 20, 2021)

Keywords: microwave imaging, near-field measurement, rough surfaces, self-adaptive dynamic differential evolution (SADDE)

We have studied the near-field measurement of periodic non-flat surfaces and compared the error between the near-field and far-field measurements. Using known boundary conditions and recorded scattering data, we derived a group of matrix functions that can be converted into an external problem, and then used the self-adaptive dynamic differential evolution (SADDE) method to recover the shape, periodic length, and dielectric constant of surfaces. We compared the search speed and stability of the surface reconstruction. The SADDE converges to the global extreme regardless of the initial guess. In a numerical simulation, even if the initial estimate was much larger than the true value, we could still find an accurate numerical solution and successfully reconstruct the surface shape function, period length, and relative dielectric constant. The simulation results show that the error in the near-field measurement is smaller than that in the far-field measurement.

\section{Introduction}

Microwave imaging uses microwaves as a transmitted signal, raising the problem of electromagnetic backscattering. The principle is to irradiate a measured object with microwaves, then use the measured external scattering field to obtain the final target. Through effective calculations, the contour and dielectric constant of objects are calculated. Remote sensing based on electromagnetic inverse scattering is a measurement technique used in microwave imaging. This technology uses microwaves to illuminate the scattering, then the reflected field is sensed using an electromagnetic sensor. This method has been widely used in telemetry, imaging of buried objects, and non-destructive testing for many years. ${ }^{(1-5)}$ Compared with other imaging technologies, the cost of equipment is lower, making this technology very valuable.

Owing to the rapid improvement of computer processor performance and the development of numerical methods, we can use a measured electromagnetic field to reconstruct the shape of a conductor or the dielectric constant of an object. Generally, a layered area is a rough surface rather than a plane surface in the real world, ${ }^{(6)}$ so a non-flat plane is more realistic and more useful in practical applications. The surface to be reconstructed can be a perfect electric *Corresponding author: e-mail: chiu@ee.tku.edu.tw https://doi.org/10.18494/SAM.2021.3282 
conductor (PEC) medium with a curved surface or two dielectric media separated by an interface. There have been many studies on PEC surface imaging problems, most of which rely on iterative algorithms. In Ref. 7, a multi-event and multi-frequency method was used to study small disturbances in three-dimensional situations. In Ref. 8, the Dirichlet and Neumann boundary conditions for the electromagnetic integral equation were given. The imaging problem of separating the rough surfaces of two dielectric media is well established. For example, the inverse problem can be solved by a semi-analytical method with the help of a perturbation series method. ${ }^{(9)}$ However, this approach can only be used for a small non-flat surface. In Ref. 10, the spectral expression for received scattered waves was used to reconstruct a lossy rough surface. However, this method is only effective for a slightly rough surface. The Kirsch-Kress method was proposed as an effective iterative method for a wide range of roughness. ${ }^{(11)}$ The basic ideas introduced in Refs. 12 and 13 enable a very comprehensive numerical analysis of rough surfaces by an iterative algorithm. In addition, microwave imaging has also been used to image buried objects in recent years. ${ }^{(14,15)}$ However, conjugate methods often become trapped at local extrema. In this paper, we compare the imaging of rough surfaces by near-field and far-field measurements using the self-adaptive dynamic differential evolution (SADDE) method. Our research is related to multivariate data analysis since many variables are involved. The rest of this paper is organized as follows: Sect. 2 covers the theoretical formulation and Sect. 3 introduces the SADDE method to solve the inverse scattering problem. Simulation results and conclusions are given in Sects. 4 and 5, respectively.

\section{Theoretical Formulation}

As shown in Fig. 1, electromagnetic waves illuminate two-dimensional periodic non-flat surfaces. Rough surfaces are separated by the half-space function $y=f(x)$. The surface has a period of $d$ in the $X$ direction and infinity in the $Z$ direction. The characteristics of the rough surface vary in the $X Y$ plane and the permittivity and permeability are represented by $\left(\varepsilon_{0}, \mu_{0}\right)$

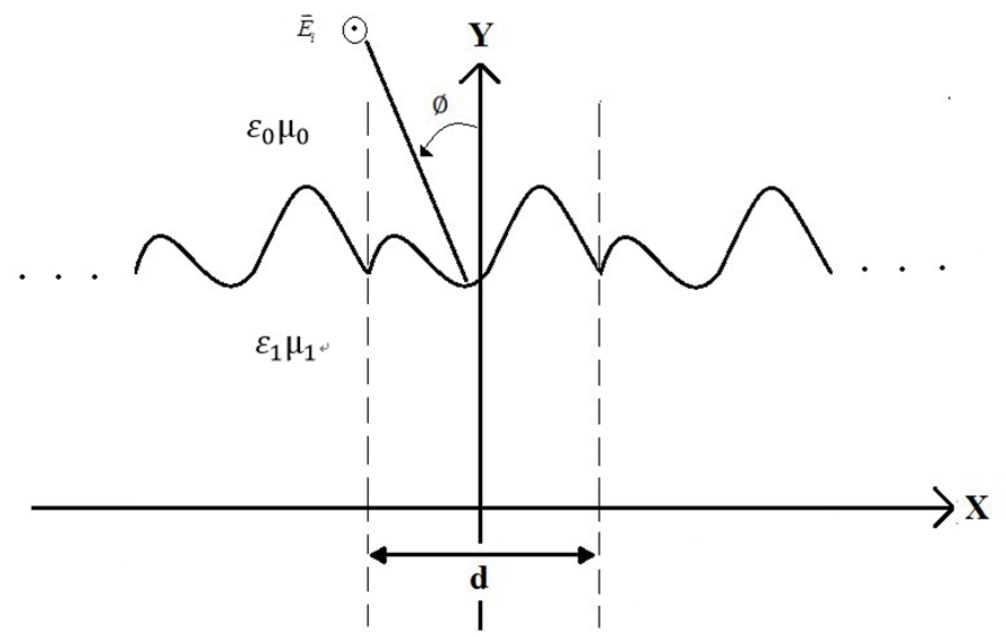

Fig. 1. Electromagnetic waves illuminating a periodic rough surface. 
and $\left(\varepsilon_{1}, \mu_{1}\right)$ for the upper and lower surfaces, respectively. We assume a TM polarized uniform plane wave $e^{j \omega t}$ with time harmonics incident along the $\mathrm{Z}$ direction. The incident electric field is represented as follows by the electric field $\vec{E}_{i}$ at the incident angle $\phi$.

$$
\vec{E}_{i}(x, y)=e^{-j k_{0}(x \cdot \sin \phi-y \cdot \cos \phi)} \hat{z}, k_{0}=\omega \sqrt{\varepsilon_{0} \mu_{0}}
$$

By Maxwell's equation for the electromagnetic field, we use the two-dimensional Green's function to express the scattered field for region $I$ as ${ }^{(16)}$

$$
\begin{aligned}
E_{S}(x, y)= & \int_{-d / 2}^{d / 2}\left[\frac{\partial G_{0}\left(x, y ; x^{\prime}, f\left(x^{\prime}\right)\right)}{\partial n^{\prime}} \cdot E_{0}\left(x^{\prime}, f\left(x^{\prime}\right)\right)-G_{0}\left(x, y ; x^{\prime}, f\left(x^{\prime}\right)\right) \cdot \frac{\partial E_{0}\left(x^{\prime}, f\left(x^{\prime}\right)\right)}{\partial n^{\prime}}\right] \\
& \cdot \sqrt{1+\left(f^{\prime}\left(x^{\prime}\right)\right)^{2}} d x^{\prime}, \text { for } y>f(x) .
\end{aligned}
$$

The electric fields on the surface and in the normal direction of the surface are expressed as $E_{0}$ and $n^{\prime}$, respectively.

$$
\begin{gathered}
G_{i}\left(x, y ; x^{\prime}, y^{\prime}\right)=\frac{1}{4 j} \sum_{l=-\infty}^{\infty} H_{0}^{(2)}\left(k_{i} \sqrt{\left(x-x^{\prime}-l d\right)^{2}+\left(y-y^{\prime}\right)^{2}}\right) \exp \left(-j k_{i} l d \sin \phi\right) \\
i= \begin{cases}0, & \text { for } y>f(x) \\
1, & \text { for } y<f(x)\end{cases}
\end{gathered}
$$

Here, $G_{i}$ is the two-dimensional periodic Green's function, $H_{0}^{(2)}$ is the second type of Hankel function, and $k_{i}$ is the wave number of the surface. The Green's function in the spectral domain is rewritten as

$$
\begin{gathered}
G_{i}\left(x, y ; x^{\prime}, y^{\prime}\right)=\sum_{l=-\infty}^{\infty} \frac{1}{2 \alpha_{l} d} \exp \left(-\alpha_{l}\left|y-y^{\prime}\right|\right) \exp \left(-j k_{l}\left|x-x^{\prime}\right|\right), \\
\alpha_{l}=\left\{\begin{array}{ll}
j \sqrt{k_{i}^{2}-k_{l}^{2}}, & k_{i}^{2}>k_{l}^{2} \\
\sqrt{k_{l}^{2}-k_{i}^{2}}, & k_{i}^{2} \leq k_{l}^{2}
\end{array}, k_{l}=\frac{2 \pi l}{d}+k_{i} \sin \phi .\right.
\end{gathered}
$$

Using Green's identity and setting the observed point on the upper and lower surfaces, we obtained following set of integral formulas:

$$
\begin{gathered}
E_{i}(x, f(x))+\int_{-d / 2}^{d / 2}\left[\frac{\partial G_{0}\left(x, f(x) ; x^{\prime}, f\left(x^{\prime}\right)\right)}{\partial n^{\prime}} \cdot E_{0}\left(x^{\prime}, f\left(x^{\prime}\right)\right)-G_{0}\left(x, f(x) ; x^{\prime}, f\left(x^{\prime}\right)\right) \cdot U_{0}\left(x^{\prime}, f\left(x^{\prime}\right)\right)\right] \\
\cdot \sqrt{1+\left(f^{\prime}\left(x^{\prime}\right)\right)^{2}} d x^{\prime}=\frac{1}{2} E_{0}(x, f(x)) .
\end{gathered}
$$


Also, by setting the observed point on the lower and upper surfaces, we obtain the following equation:

$$
\begin{gathered}
-\int_{-d / 2}^{d / 2}\left[\frac{\partial G_{1}\left(x, f(x) ; x^{\prime}, f\left(x^{\prime}\right)\right)}{\partial n^{\prime}} \cdot E_{1}\left(x^{\prime}, f\left(x^{\prime}\right)\right)-G_{1}\left(x, f(x) ; x^{\prime}, f\left(x^{\prime}\right)\right) \cdot U_{1}\left(x^{\prime}, f\left(x^{\prime}\right)\right)\right] \\
\cdot \sqrt{1+\left(f^{\prime}\left(x^{\prime}\right)\right)^{2}} d x^{\prime}=\frac{1}{2} E_{1}(x, f(x)) .
\end{gathered}
$$

Using the boundary condition with continuous tangential fields, we describe the equations as follows:

$$
\begin{gathered}
\hat{n} \times \vec{E}_{0}=\hat{n} \times \vec{E}_{1} \rightarrow E_{0}=E_{1}, \\
\hat{n} \times \vec{H}_{0}=n \times \vec{H}_{1} \rightarrow \frac{\partial E_{0}}{\partial n}=\frac{\partial E_{1}}{\partial n} .
\end{gathered}
$$

Substituting Eqs. (8) and (9) into Eqs. (6) and (7), respectively, gives

$$
\begin{aligned}
& E_{i}(x, f(x))=\frac{1}{2} E_{0}(x, f(x))-\int_{-d / 2}^{d / 2}\left[\frac{\partial G_{0}\left(x, f(x) ; x^{\prime}, f\left(x^{\prime}\right)\right)}{\partial n^{\prime}} \cdot E_{0}\left(x^{\prime}, f\left(x^{\prime}\right)\right)\right. \\
& \left.-G_{0}\left(x, f(x) ; x^{\prime}, f\left(x^{\prime}\right)\right) \cdot U_{0}\left(x^{\prime}, f\left(x^{\prime}\right)\right)\right] \cdot \sqrt{1+\left(f^{\prime}\left(x^{\prime}\right)\right)^{2}} d x^{\prime}, \\
& 0=\frac{1}{2} E_{0}(x, f(x))+\int_{-d / 2}^{d / 2}\left[\frac{\partial G_{1}\left(x, f(x) ; x^{\prime}, f\left(x^{\prime}\right)\right)}{\partial n^{\prime}} \cdot E_{0}\left(x^{\prime}, f\left(x^{\prime}\right)\right)\right. \\
& \left.-G_{1}\left(x, f(x) ; x^{\prime}, f\left(x^{\prime}\right)\right) \cdot U_{0}\left(x^{\prime}, f\left(x^{\prime}\right)\right)\right] \cdot \sqrt{1+\left(f^{\prime}\left(x^{\prime}\right)\right)^{2}} d x^{\prime} .
\end{aligned}
$$

The upper surface is obtained from the period, dielectric coefficient, and shape of the surface using Eqs. (2), (10), and (11).

When we use a numerical technique to handle imaging of the surfaces, Eqs. (2), (10), and (11) are converted into a matrix function by the method of moments. Then, through the point matching method, the impulse function is used as the basic function and the test function. Equations (10) and (11) are converted into the following matrix equations:

$$
\left[E_{i}\right]_{N \times 1}=\frac{1}{2}\left[E_{0}\right]_{N \times 1}-\left[G_{0}^{\prime}\right]_{N \times N}\left[E_{0}\right]_{N \times 1}+\left[G_{0}\right]_{N \times N}\left[U_{0}\right]_{N \times 1},
$$




$$
[0]_{N \times 1}=\frac{1}{2}\left[E_{0}\right]_{N \times 1}+\left[G_{1}^{\prime}\right]_{N \times N}\left[E_{0}\right]_{N \times 1}-\left[G_{1}\right]_{N \times N}\left[U_{0}\right]_{N \times 1}
$$

and

$$
\begin{gathered}
{\left[G_{i}^{\prime}\right]_{m n}=\frac{\partial G_{i}\left(x_{m}, f\left(x_{m}\right) ; x_{n}^{\prime}, f\left(x_{n}^{\prime}\right)\right)}{\partial n^{\prime}} \cdot \sqrt{1+\left(f^{\prime}\left(x_{n}^{\prime}\right)\right)^{2}} \cdot \Delta x^{\prime},} \\
{\left[G_{i}\right]_{m n}=G_{i}\left(x_{m}, f\left(x_{m}\right) ; x_{n}^{\prime}, f\left(x_{n}^{\prime}\right)\right) \cdot \sqrt{1+\left(f^{\prime}\left(x_{n}^{\prime}\right)\right)^{2}} \cdot \Delta x^{\prime} \quad i=\left\{\begin{array}{ll}
0, & \text { for } y>f(x) \\
1, & \text { for } y<f(x)
\end{array} .\right.}
\end{gathered}
$$

Here, $m$ and $n$ are the indices for the observation and source, respectively. The forward scattering problem is solved by solving Eqs. (12) and (13) to obtain the matrices of $\left[E_{0}\right]$ and $\left[U_{0}\right]$. The scattering field can be calculated using Eq. (2), with the following matrix representation:

$$
\left[E_{S}\right]_{M \times 1}=\left[G_{0}^{\prime}\right]_{M \times N}\left[E_{0}\right]_{N \times 1}-\left[G_{0}\right]_{M \times N}\left[U_{0}\right]_{N \times 1},
$$

where $M$ is the total number of observation points. The recorded point is set above the surface.

\section{Inverse Scattering}

We used Eqs. (10) and (11) to compute $E_{0}$ and $U_{0}$ from the estimated period length, dielectric coefficient, and shape function of the surface, then calculated the scattered field through Eq. (2). To monitor the iteration process, we define the objective function as

$$
O B J F=\frac{1}{M}\left\{\sum_{m=1}^{M}\left[E_{s}^{\text {meas }}\left(\vec{r}_{m}\right)-E_{s}^{c a l}\left(\vec{r}_{m}\right)\right]^{2} /\left[E_{s}^{\text {meas }}\left(\vec{r}_{m}\right)\right]^{2}\right\}^{\frac{1}{2}},
$$

where $E_{s}^{c a l}\left(\vec{r}_{m}\right)$ is the optimized scattering field and $E_{s}^{\text {meas }}\left(\vec{r}_{m}\right)$ is the measured scattering field. A smaller OBJF indicates a better estimate.

SADDE is a modified version of the dynamic DDE. The SADDE algorithm adds a mechanism to dynamically change the adjustment factor during the search process. First, the algorithm initializes the population parameters randomly to compute the objective function. Second, we mutate the test vector according to the control vector and adjust the control vector for the next generation, and consider whether to use the crossover mechanism according to the preset probability. If we use this mechanism, the new crossover particle is compared with the previous target value, and then the global best particle is updated.

The flow of the SADDE method is shown in Fig. 2. The parameter vector is evolving in the $g$ th generation and can be expressed as 


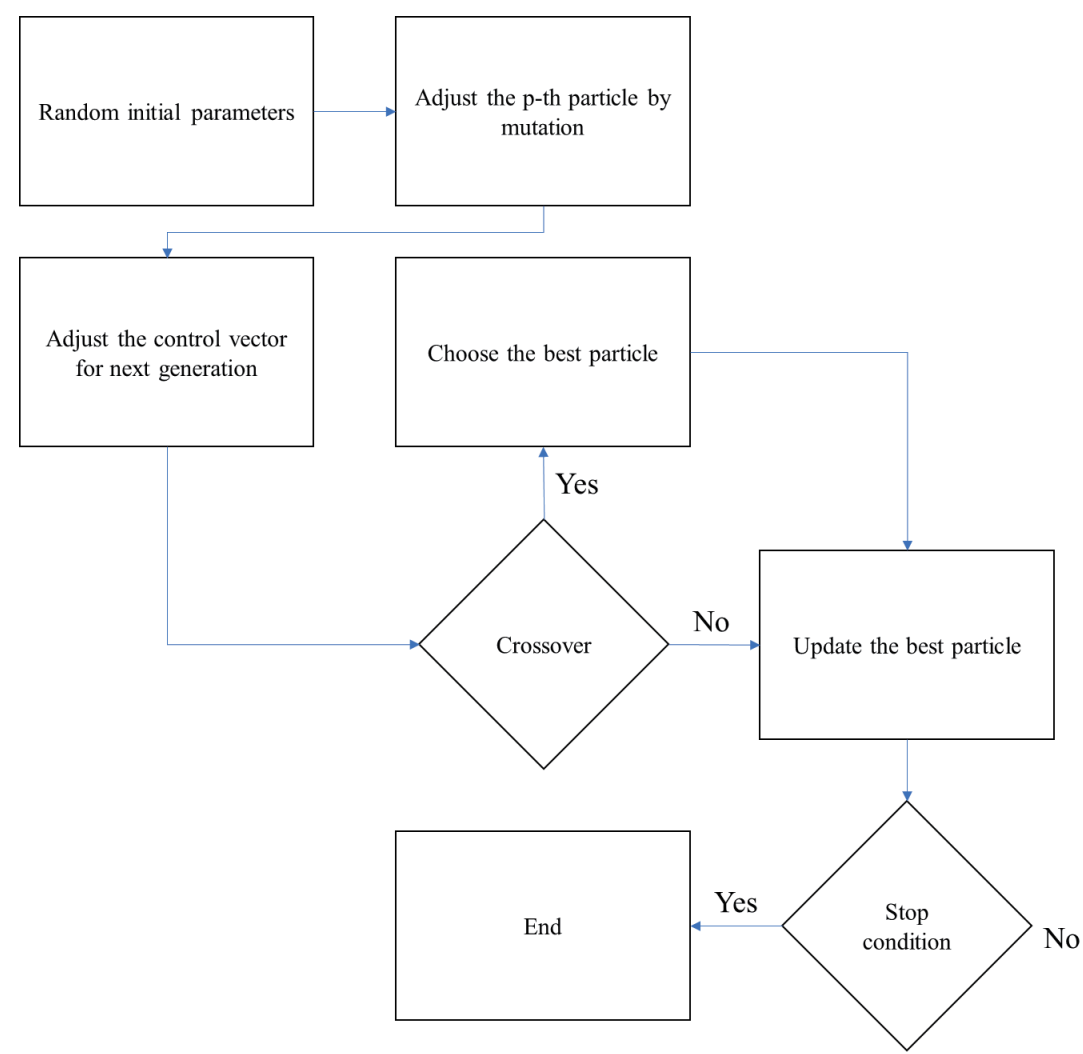

Fig. 2. Flow of the SADDE method.

$$
X_{j}^{g}=\left[x_{j 1}, x_{j 2}, x_{j 3}, \ldots, x_{j D}\right], j=1,2, \ldots, N_{p},
$$

where $N_{p}$ is the total number of vectors and $D$ is number of parameters in each vector. The mutation process is expressed as

$$
V_{j}^{g+1}=X_{j}^{g}+F_{1} \cdot\left(X_{b e s t}-X_{j}^{g}\right)+F_{2} \cdot\left(X_{r 1}^{g}-X_{r 2}^{g}\right), r_{1}, r_{2} \in\left[1, N_{p}\right] \& r_{1} \neq r_{2},
$$

where $F_{1}$ and $F_{2}$ are scaling factors. Next, for the given crossover rate, we use a crossover to find the new parameter vectors and we keep the best vector in the iterative process. The final best vector is the solution of the imaging problem.

\section{Numerical Results}

Consider a uniform plane wave with an amplitude of unity incident from above the surface with an incident angle of $\phi=35^{\circ}$ and a frequency of $3 \mathrm{GHz}$, that is, the wavelength is $0.1 \mathrm{~m}$. The upper surface is air, that is, $\varepsilon_{0}=8.854 \times 10^{-12} \mathrm{~F} / \mathrm{m}$, and the dielectric coefficient below the surface is $\varepsilon_{1}=2.56 \varepsilon_{0}$. The period length is $d=0.083 \mathrm{~m}$. The measuring points are arranged 
equidistantly on a straight line from $x=-0.03 \mathrm{~m}$ to $0.03 \mathrm{~m}$ with nine measuring positions, as shown in Fig. 1.

In the following simulation, the number of terms $M_{d}$ used to expand the surface current in the forward scattering problem is 50 . The calculated number of unknowns is $N_{f}$ for the surface expanded by the Fourier function, with one unknown period length $d$ and one unknown relative permittivity $\varepsilon_{1}$. In other words, there are $\left(N_{f}+2\right)$ unknowns. The number of particles is 75 and the number of generations is 500 . The shape of the non-flat surface function expanded by the Fourier series is

$$
F(x)=\sum_{1}^{N_{f} / 2} a_{n} \cos \left(n \cdot \frac{2 \pi x}{d}\right)+\sum_{N_{f} / 2+1}^{N_{f}} a_{n} \sin \left(\left(n-\frac{N_{f}}{2}\right) \cdot \frac{2 \pi x}{d}\right) .
$$

$N_{f}$ is 6 for the following simulation.

The errors for the dielectric constant, period length, and shape are computed using the following equations:

$$
\begin{gathered}
D E P S=\frac{\left|\varepsilon^{c a l}-\varepsilon^{\text {exact }}\right|}{\varepsilon^{\text {exact }}}, \\
D P=\frac{\left|d^{\text {cal }}-d^{\text {exact }}\right|}{d^{\text {exact }}}, \\
D F=\frac{1}{N_{D}}\left\{\sum_{1}^{N_{D}}\left[F^{\text {exact }}(x)-F^{c a l}(x)\right]^{2} /\left[F^{\text {exact }}(x)\right]^{2}\right\}^{\frac{1}{2}} .
\end{gathered}
$$

Example 1 is a convex non-flat periodic surface with the shape function

$$
F(x)=0.03 \cos \left(\frac{2 \pi x}{d}\right)+0.006 \cos \left(\frac{6 \pi x}{d}\right)+0.007 \sin \left(\frac{4 \pi x}{d}\right)+0.005 \sin \left(\frac{6 \pi x}{d}\right) .
$$

The surface reconstruction results for 10\% noise are drawn in Fig. 3, where Figs. 3(a) and 3(b) show the results for near-field $(y=0.05 \mathrm{~m})$ and far-field $(y=2 \mathrm{~m})$ measurements, respectively. The reconstruction of the final generation in the near field almost fits the actual value, while that in the far field has some deviation from the actual value. Figure 4 shows a comparison of DP and DF for near-field and far-field measurements with $10 \%$ noise. The near field converges to the extreme values of DP and DF in about 200 generations as shown in Fig. 4(a). From Fig. 4(b), although the far-field part also converges, the convergence value is worse than that of the near- 


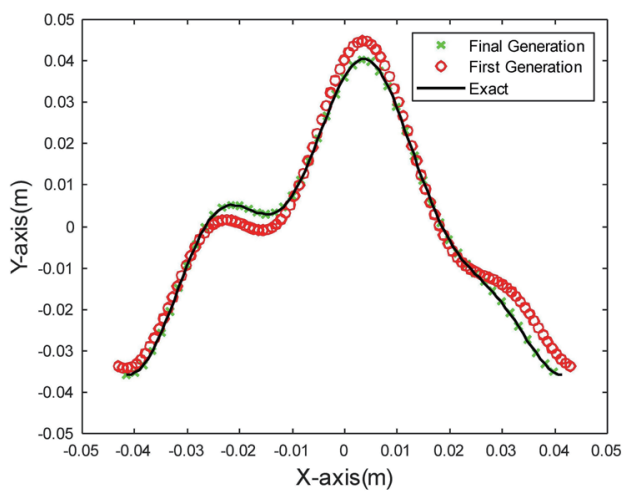

(a)

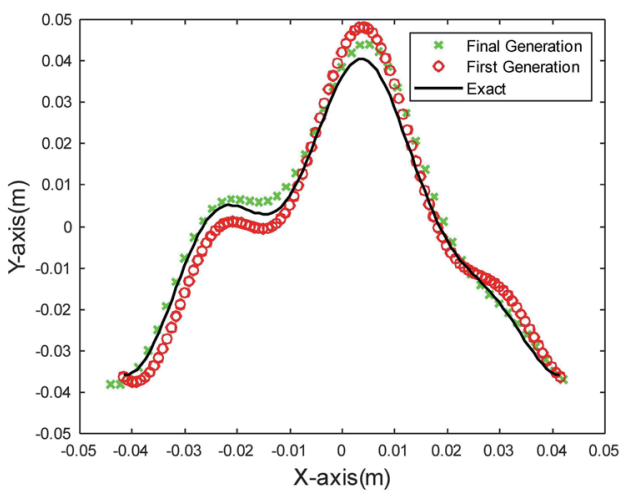

(b)

Fig. 3. (Color online) (a) Reconstruction of the surfaces of the first example for near-field measurement. (b) Reconstruction of the surfaces of the first example for far-field measurement.

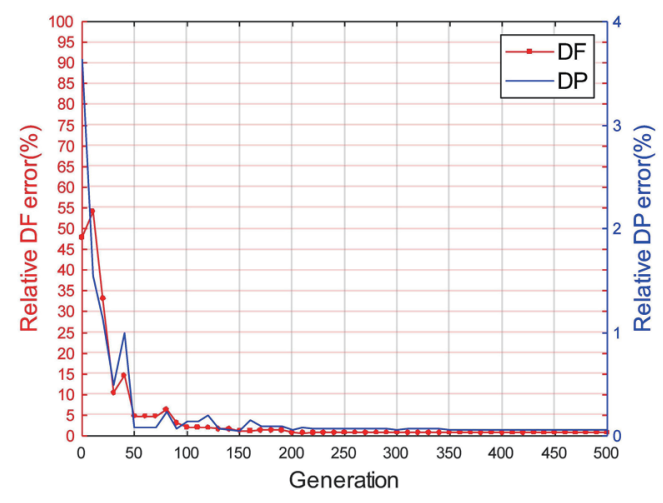

(a)

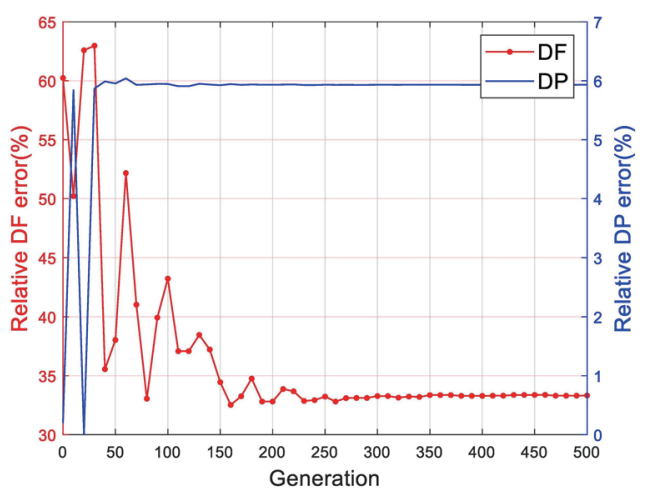

(b)

Fig. 4. (Color online) (a) DP and DF of the first example for near-field measurement. (b) DP and DF of the first example for far-field measurement.

field measurement. DF and DEPS are plotted in Fig. 5 for 10\% noise. It can be seen that the error value in the near field [Fig. 5(a)] is much smaller than that in the far field [Fig. 5(b)].

To test the robustness of the algorithm to noise, different noise levels were added in the scattered field. Figures 6(a) and 6(b) show a comparison of the results of near-field and far-field measurements, respectively, for $0.1,1$, and $10 \%$ noise levels. It can be seen that the near-field DF, DP, and DEPS are always better than the far-field values, so the near field has better robustness to noise.

The following concave surface is chosen for the second example:

$$
F(x)=0.02 \cos \left(\frac{2 \pi x}{d}\right)+0.02 \cos \left(\frac{6 \pi x}{d}\right)+0.003 \sin \left(\frac{4 \pi x}{d}\right)+0.002 \sin \left(\frac{6 \pi x}{d}\right)
$$




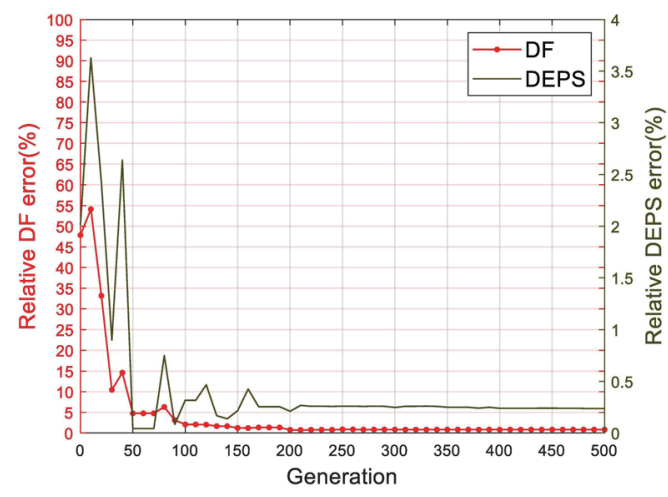

(a)

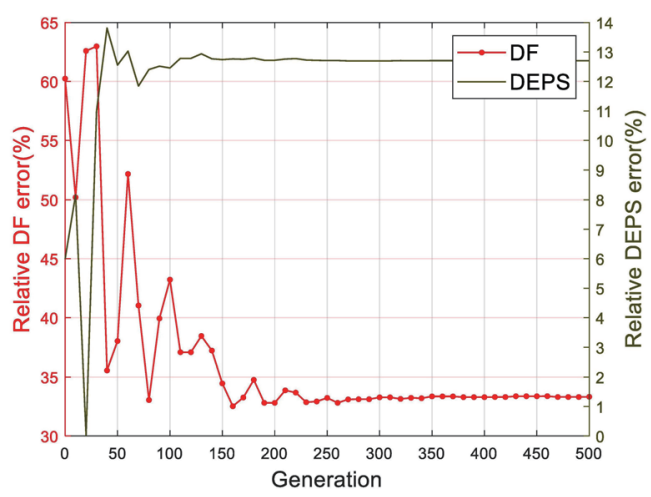

(b)

Fig. 5. (Color online) (a) DF and DEPS of the first example for near-field measurement. (b) DF and DEPS of the first example for far-field measurement.

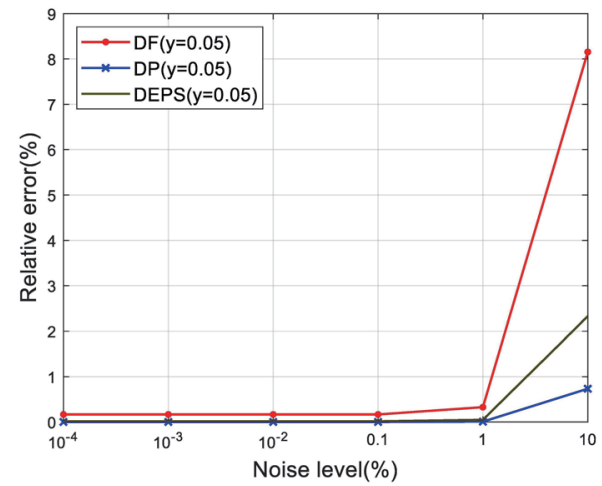

(a)

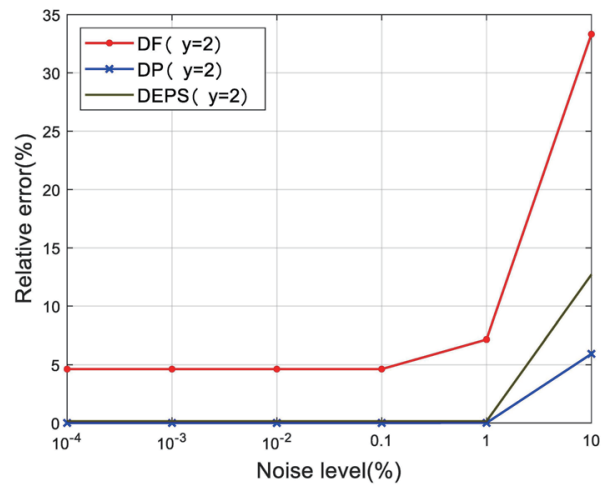

(b)

Fig. 6. (Color online) (a) Error parameters versus noise level of example 1 for near-field measurement. (b) Error parameters versus noise level of example 1 for far-field measurement.

Figures 7(a) and 7(b) show a comparison of the results of reconstruction by near-field and farfield measurements with $10 \%$ noise, respectively. It can be observed that even if there is a gap between the initial estimate and the correct value, good reconstruction results can still be obtained for near-field measurement by the SADDE method. It is clear that the reconstruction obtained by far-field measurement is inferior to that obtained by near-field measurement.

In the next example, the following shape function has multiple undulations:

$$
F(x)=0.02 \cos \left(\frac{4 \pi x}{d}\right)+0.003 \sin \left(\frac{4 \pi x}{d}\right)+0.02 \sin \left(\frac{6 \pi x}{d}\right) .
$$

Figure 8 is the surface reconstruction result. The shape for the near-field measurement is clearly better than that for the far-field measurement and almost coincides with the actual 


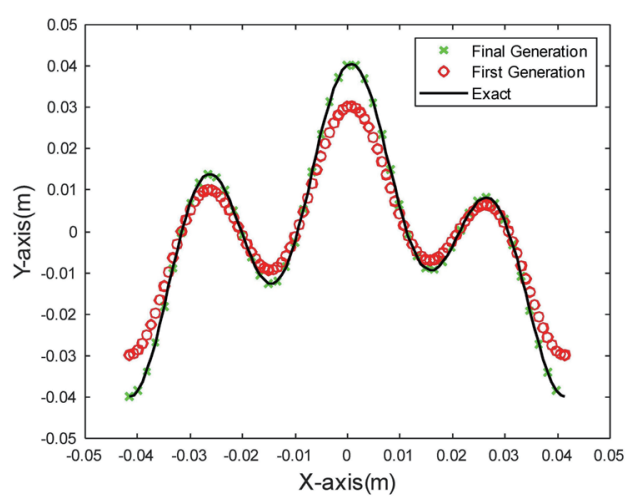

(a)

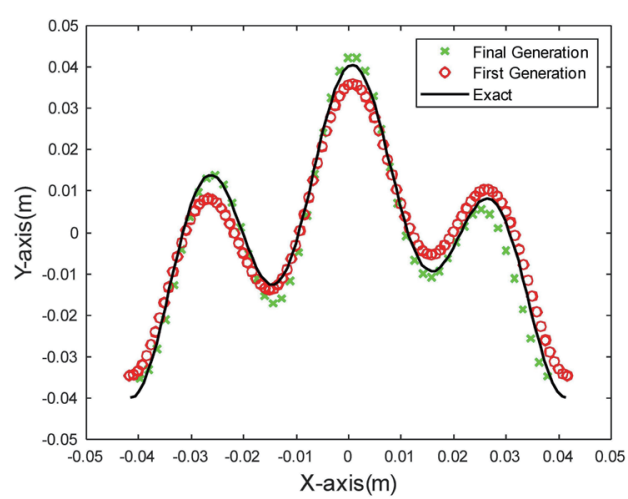

(b)

Fig. 7. (Color online) (a) Reconstructed surfaces of example 2 for near-field measurement. (b) Reconstructed surfaces of example 2 for far-field measurement.

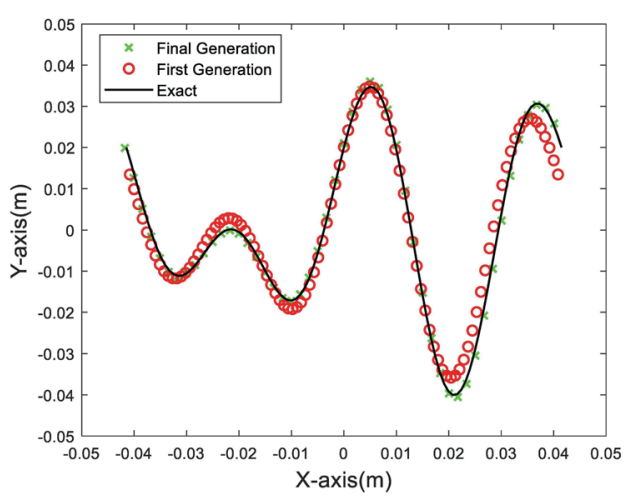

(a)

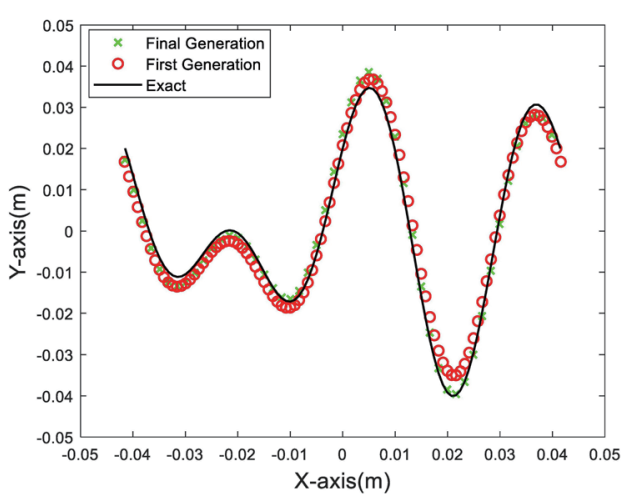

(b)

Fig. 8. (Color online) (a) Reconstructed surfaces of example 3 for near-field measurement. (b) Reconstructed surfaces of example 3 for far-field measurement.

surface. The simulation results of the three examples show that our algorithm has good reconstruction results.

\section{Conclusion}

In this article, we discuss the application of inverse scattering to non-flat surfaces. Rigorous theories are used to derive nonlinear integral equations, then non-flat surface problems are transformed into optimization problems. To avoid the problem of easily falling into local minima, we use the SADDE method, and we successfully reconstructed surfaces with different shapes, period lengths, and dielectric constants by numerical simulations.

The numerical simulations show that the SADDE method can find the best solution, confirming that this evolution method is suitable for complex optimization problems. From graphs showing the errors of the surface shape, period length, and dielectric constant, it is found 
that the error is lower in near-field measurement than in far-field measurement. Effective reconstruction and robustness to noise were shown for not only the concave and convex surfaces but also surfaces with multiple undulations. Similarly, the reconstruction effect is better in the near field than in the far field for all surfaces.

The research reported in this paper still has a lot of room for development. It can be applied to multilayered spaces and buried objects. In addition, we only discuss the case of plane waves. It is possible to extend this method to cylindrical waves.

\section{References}

1 A. Randazzo, C. Ponti, A. Fedeli, C. Estatico, P. D’Atanasio, M. Pastorino, and G. Schettini: IEEE Trans. Geosci. Remote Sens. Early Access (2021) 1.

2 C. Yang, J. Zhang, and M. S. Tong: IEEE Trans. Antennas Propag. 69 (2021) 1078.

3 Y. Huang, R. Song, K. Xu, X. Ye, C. Li, and X. Chen: IEEE Sens. J. 21 (2021) 4900.

4 Y. Gan, C. Yin, Q. Fan, and A. Li: IEEE Access 8 (2020) 195122.

5 H. I. Azeez, W. S. Chen, C. K. Wu, C. M. Cheng, and H. C. Yang: Sens. Mater. 30 (2018) 595.

6 G. Jun and W. Xiaobing: Proc. 2014 3rd Asia-Pacific Conf. (Asia-Pacific, 2014) 987-990.

7 M. El-Shenawee and E. L. Miller: IEEE Trans. Geosci. Remote Sens. 42 (2004) 2499.

8 Y. Chen, O. R. Spivack, and M. Spivack: Inver. Problems 34 (2018) 1.

9 G. Bao and P. Li: SIAM J. Imag. Sci. 7 (2014) 867.

10 I. Akduman, R. Kress, and A. Yapar: Inver. Problems 22 (2006) 939.

11 J. Li, G. Sun, and B. Zhang: App. Anal. 96 (2016) 85.

12 A. Sefer and A. Yapar: IEEE Trans. Geosci. Remote Sens. Early Access (2020) 1.

13 C. C. Chiu and M. K. Chan: Micro. Opt. Technol. Lett. 60 (2018) 1719.

14 C. C. Chiu, B. J. Hong, and J. Hao: J. Elec. Waves Appl. 34 (2020) 1015.

15 C. C. Chiu, G. Z. Lee, J. Hao, and B. J. Hong: J. Elec. Waves Appl. 33 (2019) 1905.

16 A. Ishimaru: Electromagnetic Wave Propagation, Radiation, and Scattering (New Jersey, Prentice-Hall, 1991).

\section{About the Authors}

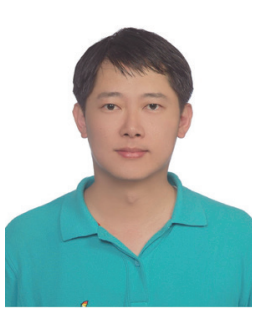

Wei Chien was born in Taipei, Taiwan, Republic of China, on April 21, 1974. He received his B.S.C.E. degree from Ta Tung University, Taipei, Taiwan, in 1997 and his M.S.E.E. degree from Tamkang University, Taipei, in 1999. From 1999 to 2001, he served in the ROC Army Force and was an assistant in the Department of Electrical Engineering. From 2007 to 2014, he was an assistant professor in the Department of Electronic Engineering, De Lin Institute of Technology. From 2014 to 2017, he was a professor in the Department of Computer Engineering, Ningde Normal University. Since 2017, he has been a professor in the School of Electronic and Information Engineering, Beibu Gulf University. His research area is deep learning for inverse scattering. (air180@seed.net.tw) 


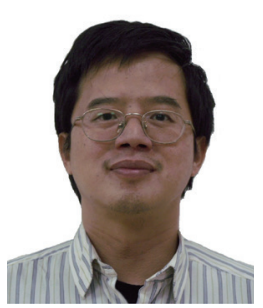

Chien-Ching Chiu received his B.S.C.E. degree from National Chiao Tung University, Hsinchu, Taiwan, in 1985 and his M.S.E.E. and Ph.D. degrees from National Taiwan University, Taipei, in 1987 and 1991, respectively. From 1987 to 1989, he was a communication officer with the ROC Army Force. In 1992, he joined the Department of Electrical Engineering, Tamkang University, where he is now a professor. From 1998 to 1999, he was a visiting scholar at Massachusetts Institute of Technology, Cambridge, and the University of Illinois at Urbana-Champaign, USA. He was a visiting professor at the University of Wollongong, Australia, in 2006. Moreover, he was a visiting professor at the University of London, United Kingdom, in 2011. From 2019 to 2020, he was a visiting professor at University Tunku Abdul Rahman, Malaysia. His current research interests include inverse problems, deep learning for microwave imaging, indoor wireless communications, and simultaneous wireless information and power transfer systems. He has published more than 150 journal papers on inverse scattering problems, communication systems, and optimization algorithms. (chiu@ee.tku.edu.tw)

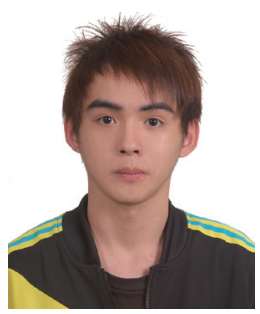

Po-Hsiang Chen received his B.S. degree from HungKuo Delin University of Technology, Tucheng, Taiwan, in 2017. Since 2018, he has been working toward his M.S. degree at the Department of Electrical and Computer Engineering, Tamkang University, Taiwan. His research areas are inverse scattering and deep learning. (kic0606@gmail.com)

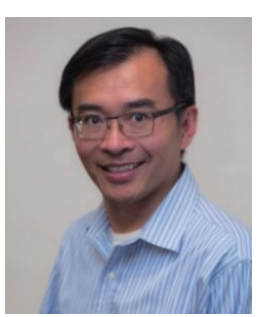

Hao Jiang received his B.S. degree in materials science from Tsinghua University, China, in 1994 and his Ph.D. degree in electrical engineering from the University of California, San Diego, in 2000. He has been with San Francisco State University (SFSU) since August 2007. He is a professor in electrical and computer engineering. Prior to joining SFSU, he worked for Broadcom Corporation, Jazz Semiconductor, and Conexant Systems Inc. His research interest is the development of low-power, high-speed, energyefficient, and small-form-factor integrated circuit systems for biomedical and bio-inspired computing systems. (jianghao@sfsu.edu)

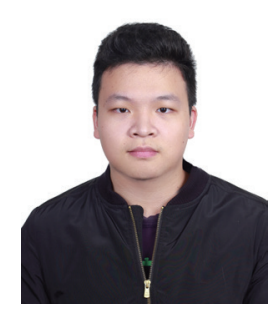

Shun-Jie Chan received his Master's degree from the Department of Electrical and Computer Engineering, Tamkang University, Taiwan, in 2020. His research interests include inverse scattering, electromagentic field measurement, and differential evolution. (jacky30116@gmail.com) 